\title{
Enhanced Visual Motion Perception in Major Depressive Disorder
}

\author{
Julie D. Golomb, ${ }^{1}$ Jenika R. B. McDavitt, ${ }^{2}$ Barbara M. Ruf, ${ }^{3}$ Jason I. Chen, ${ }^{3}$ Aybala Saricicek, ${ }^{3}$ Kathleen H. Maloney, ${ }^{3}$ \\ Jian $\mathrm{Hu}^{3}$ Marvin M. Chun, ${ }^{1,2}$ and Zubin Bhagwagar ${ }^{3}$ \\ ${ }^{1}$ Interdepartmental Neuroscience Program and ${ }^{2}$ Department of Psychology, Yale University, and ${ }^{3}$ Department of Psychiatry, Yale University School of \\ Medicine, New Haven, Connecticut 06520
}

\begin{abstract}
Major depressive disorder (MDD) is a mood disorder that is not traditionally considered to affect the visual system. However, recent findings have reported decreased cortical levels of the inhibitory neurotransmitter GABA in occipital cortex. To explore possible functional consequences of MDD on visual processing, we applied a psychophysical visual motion processing task in which healthy young adults typically exhibit impaired perceptual discrimination of large high-contrast stimuli. It has been suggested that this phenomenon, spatial suppression, is mediated by GABAergic center-surround antagonism in visual pathways. Based on previous findings linking MDD to occipital GABA dysfunction, we hypothesized that MDD patients would exhibit decreased spatial suppression, leading to the counterintuitive hypothesis of better psychophysical performance. Indeed, motion perception for typically suppressed stimuli was enhanced in patients with MDD compared with age-matched controls. Furthermore, the degree of spatial suppression correlated with an individual's illness load; patients with greater lifetime duration of depression exhibited the least spatial suppression and performed the best in the high-contrast motion discrimination task. Notably, this decrease in spatial suppression persisted beyond recovery and without the confound of acute illness or treatment; all patients had been clinically recovered and unmedicated for several months at the time of testing, suggesting that depression has ubiquitous consequences that may persist long after mood symptoms have receded. This finding raises the possibility that spatial suppression may represent a sensitive endophenotypic marker of trait vulnerability in MDD.
\end{abstract}

\section{Introduction}

Major depressive disorder (MDD) is the one of the world's leading causes of disability, affecting 151 million people worldwide (World Health Organization, 2008). While there has been enormous interest in neurotransmitter systems like the monoamines in MDD, there has also been a recent resurgence of interest in the major inhibitory neurotransmitter GABA and its role in mood disorders (Emrich et al., 1980; Lloyd et al., 1989; Petty, 1995; Shiah and Yatham, 1998). Over the years, converging lines of evidence have suggested a dysfunctional GABAergic system in MDD (Petty et al., 1992; Brambilla et al., 2003; Kalueff and Nutt, 2007). The application of recent advances in proton magnetic resonance spectroscopy (MRS) has demonstrated persistent dys-

\footnotetext{
Received Feb. 27, 2009; revised June 1, 2009; accepted June 11, 2009

This work was supported by research grants from the National Institutes of Health (Grants R01-EY014193 and P30-EY000785 to M.M.C., K23-MH077914 to Z.B., and F31-MH083374 to J.D.G.), the National Science Foundation (Graduate Research Fellowship to J.D.G.), the National Alliance for Research on Schizophrenia and Depression (Z.B.), and the Clinical and Translational Science Award Grant UL1 RR024139 from the National Center for Research Resources to Yale University (Z.B.). We acknowledge support from the Clinical Neuroscience Research Unit at the Connecticut Mental Health Center and the Connecticut Department of Mental Health and Addictions Services, and we thank A. Leber and N. Turk-Browne for helpful comments and discussion.

Correspondence should be addressed to either Julie Golomb or Marvin M. Chun, Department of Psychology, Yale University, P.0. Box 208205, New Haven, CT 06520-8205, E-mail: julie.golomb@yale.edu or marvin.chun@yale.edu; or Zubin Bhagwagar, Clinical Neuroscience Research Unit, Abraham Ribicoff Research Facilities, Connecticut Mental Health Center, 34 Park Street, New Haven, CT 06519. E-mail: zubin.bhagwagar@yale.edu.

J. R. B. McDavitt's present address: Department of Psychology, University of Illinois, Urbana-Champaign, Champaign, IL 61820 .

D0I:10.1523/JNEUROSCI.1003-09.2009

Copyright $\odot 2009$ Society for Neuroscience $\quad$ 0270-6474/09/299072-06\$15.00/0
}

function of GABA in the occipital cortex of unmedicated acutely depressed patients; GABA levels are significantly decreased in patients with MDD compared with healthy controls (Sanacora et al., 1999, 2004). Although acute or chronic treatment can ameliorate the deficit in occipital GABA (Sanacora et al., 2002, 2003; Bhagwagar et al., 2004), these cortical GABAergic deficits persist even in asymptomatic, medication-free patients with MDD (Bhagwagar et al., 2007, 2008).

However, the functional consequences, if any, of the observed deficit in occipital cortical GABA in MDD remain unclear. Many fundamental properties of visual processing, such as centersurround antagonism, rely on inhibitory connections between neurons in the occipital cortex that are likely mediated by GABA (Tsumoto et al., 1979; Angelucci and Bullier, 2003; Lee and Zhou, 2006; Schwabe et al., 2006). Given the critical role of GABAergic inhibition in the visual system, we hypothesized that the reported dysfunction of occipital GABA in MDD may have significant consequences for visual perception.

We used a perceptual task designed to be sensitive to inhibitory processes in visual cortex (Tadin et al., 2003). In this task, participants view drifting sinusoidal gratings of varying size and contrast and attempt to discriminate their direction of motion (Fig. 1). In healthy young adults, increasing the size of a lowcontrast stimulus results in improved motion discrimination performance, reflecting the phenomenon of spatial summation. On the other hand, increasing the size of a high-contrast stimulus decreases performance, a counterintuitive effect known as spatial suppression (Tadin et al., 2003). Spatial suppression only occurs 


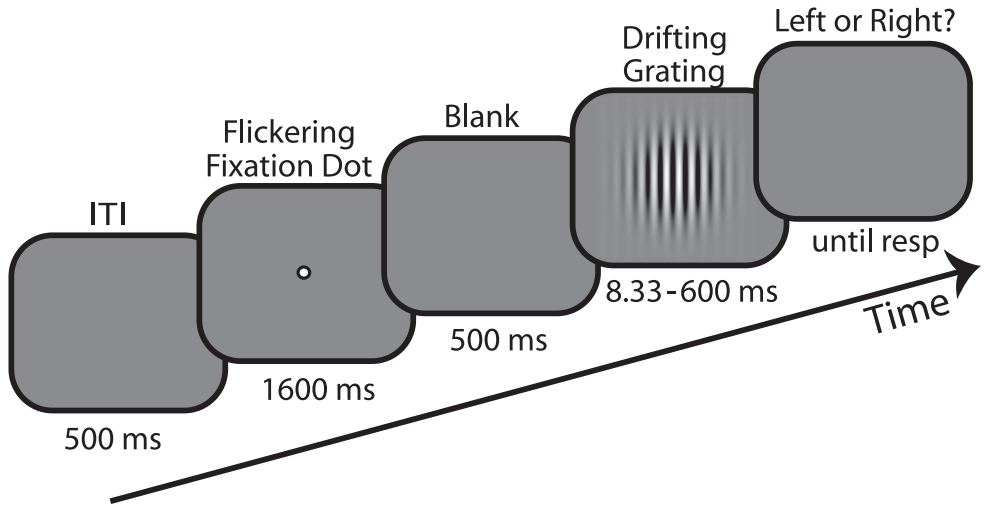

Figure 1. Experimental task. Each trial began with a flickering black and white fixation dot for $1600 \mathrm{~ms}$, followed by a $500 \mathrm{~ms}$ blank screen. A sinusoidal grating of either high ( $92 \%)$ or low $(2.8 \%)$ contrast and small $\left(0.7^{\circ}\right)$ or large $\left(5.0^{\circ}\right)$ size then appeared in the center of the screen. The grating drifted at a rate of $2 \% \mathrm{~s}$ to either the left or the right; the duration of the drift was staircased to achieve $\sim 77 \%$ accuracy (Fig. 2). Minimum duration was set at a single $120 \mathrm{~Hz}$ frame refresh $(8.33 \mathrm{~ms}$ ); no maximum was set, but duration did not surpass $600 \mathrm{~ms}$ for any subject. Once the grating disappeared, the subject made a two-alternative forced-choice response discriminating the direction of motion. The next trial began $500 \mathrm{~ms}$ after the response. Trials were blocked by condition, with each block containing 150 trials. ITI, Intertrial interval.

Table 1. Demographic data for healthy control and recovered depressed groups

\begin{tabular}{lccl}
\hline & Healthy controls & Recovered depressed & $p$ (one-way ANOVA) \\
\hline Gender & 7 male, 9 female & 7 male, 9 female & 1.00 \\
Age (years) & $21.4(2.00)$ & $23.2(4.25)$ & 0.15 \\
Decimal visual acuity & $1.71(0.31)$ & $1.65(0.35)$ & 0.59 \\
Contrast sensitivity & $172.2(48.9)$ & $163.8(63.3)$ & 0.67 \\
WRAT percentile & $83.1(12.3)$ & $86.8(14.8)$ & 0.46 \\
HAM-D & $1.07(1.58)$ & $2.44(2.31)$ & $0.07^{*}$
\end{tabular}

Mean (SD) for each group and significance level for between-group statistics. *Note that although the HAM-D difference trended toward significance, both groups were well within the euthymic range $(<7)$.

at high contrast (Pack et al., 2005; Paffen et al., 2005) and is thought to reflect center-surround antagonism in neurons in the middle temporal visual area (MT or V5) (Pack et al., 2005; Tadin and Lappin, 2005), which receives direct input from occipital visual areas. Similar paradoxical contrast-dependent effects have also been found for both behavioral performance and MT neuron activity during other types of motion processing tasks (Seitz et al., 2008).

Interestingly, high-contrast spatial suppression is reduced in certain populations, including older adults (Betts et al., 2005) and patients with schizophrenia (Tadin et al., 2006), presumably because of decreased GABAergic interactions. Hence, we hypothesized that patients with MDD would show decreased spatial suppression-that is, relatively better motion discrimination performance for large, high-contrast stimuli-based on previous neurobiological evidence directly linking this disorder with occipital GABA deficits. It should be noted that the GABAergic hypothesis predicts this counterintuitive increase in motion perception only for large, high-contrast stimuli that are typically suppressed; stimuli that would typically benefit from GABAergic center-surround modulation (such as small stimuli requiring fine discriminations) could presumably be impaired in the same population.

\section{Materials and Methods}

Participants. The interpretation of studies of acutely depressed subjects can be confounded by the epiphenomenon of acute illness, treatment effects, withdrawal of treatment effects, or some combination of these factors. To avoid these confounding effects, we recruited a group of fully recovered, euthymic, and unmedicated subjects with a diagnosis of recurrent familial MDD, and a group of never-depressed healthy control subjects matched for age, gender, IQ, visual acuity, and contrast sensitivity.

Subjects were native English speakers aged $18-35$ years with no current or past history of serious medical or neurological illness, other than described below, and no significant current or past alcohol or illicit substance dependence or abuse. Informed consent was obtained from all subjects, and the study protocol was approved by the Human Investigation Committee of the School of Medicine at Yale University. Subjects were compensated with a monetary sum for their time.

All subjects were screened using the Structured Clinical Interview for DSM-IV Disorders (SCID) (First et al., 1997) and the Hamilton Rating Scale for Depression (21-item version, HAM-D) (Hamilton, 1960). Healthy control subjects had no current or past history of any psychiatric disorder, and reported having no first-degree relatives with any Axis I psychopathology. Recovered MDD patients had experienced at least two episodes of major depression in the past, as defined by the DSM-IV, and reported having at least one first-degree relative who had a lifetime history of depression. All patients had an early onset major depressive disorder (age of onset $<21$ ), and patients with any comorbid Axis I disorders were excluded. All patients had been medication-free and euthymic for a minimum of 4 months to meet criteria for recovery as per guidelines (Rush et al., 2006). Subjects were rated to be recovered from depression on four criteria: self-reported recovery, the absence of clinically relevant symptoms during a clinical interview with an experienced psychiatrist, absence of criterion for a major depressive episode judged by the SCID, and a score of $<7$ on the HAM-D. The psychiatric screening was conducted independently of the perceptual experiments; the psychiatrist had no knowledge of the subject's behavioral performance on the task.

To address other factors that might affect task performance, all subjects were run in a series of screening tasks measuring visual acuity, visual contrast sensitivity, and IQ. Subjects' visual acuity and contrast sensitivity were measured using the Freiburg Visual Acuity and Contrast test (FrACT) (Bach, 1996), which was administered at a viewing distance of $200 \mathrm{~cm}$. Premorbid IQ was measured using the Wide Range Achievement Test (WRAT) (Wilkinson, 1993) reading subtest. Subjects who did not meet our predefined exclusion criteria for these measures were considered ineligible for the experiment; exclusion criteria included an inability to display normal or corrected-to-normal vision, as measured by a visual acuity score $>1.0$, an inability to resolve low-contrast stimuli ( $2 \%$ contrast or less), measured by a contrast sensitivity threshold score of $>50$, and a score $<50 \%$ for the standardized WRAT percentile. Subjects who did not meet the clinical criteria outlined above were also ineligible for the experiment.

A total of 17 recovered depressed patients and 24 healthy control subjects met the eligibility requirements for this experiment; we intentionally oversampled the control population to allow for post hoc age and gender matching with the patient group. Two control subjects and one recovered depressed patient were excluded from analyses because we could not obtain duration thresholds for at least one of the four conditions using the Weibull function fitting method (see below); an additional control subject was excluded because the Weibull fits for at least one condition produced threshold values that were statistical outliers (greater than three SDs outside the mean).

To match the remaining 16 recovered depressed patients, we selected 16 healthy control subjects, so that the two groups did not differ significantly for age or gender (Table 1). Had we included the full set of control subjects, there would have been a significant difference in age, but the pattern of threshold and suppression results reported here would have otherwise remained intact (supplemental figure and analysis, available at www.jneurosci.org as supplemental material).

Experimental setup. Stimuli were generated on a Macintosh G4 com- 
puter (Apple Computers) using the Psychtoolbox extension (Brainard, 1997) for Matlab (MathWorks) and were presented on a linearized 17 inch flat-screen cathode-ray tube monitor $(800 \times 600$ pixel resolution, $120 \mathrm{~Hz})$. Subjects were seated at a chin rest positioned 127 $\mathrm{cm}$ from the monitor, so that the entire display subtended $17.1 \times 12.8^{\circ}$ of visual angle. The experiment was conducted in a darkened room, with the monitor serving as the only source of light. Mean luminance was $55.2 \mathrm{~cd} /$ $\mathrm{m}^{2}$. Participants made keypress responses on a standard Macintosh keyboard.

Stimuli. Stimuli were 1 cycle per degree drifting vertical sine wave gratings surrounded by two-dimensional Gaussian envelopes. Stimulus size (defined as twice the SD of the envelope) was either $0.7^{\circ}$ or $5.0^{\circ}$, and stimulus contrast was either $2.8 \%$ or $92 \%$ [defined as $\left(L_{x}-\right.$ $\left.L_{\text {ave }}\right) / L_{\text {ave }}$, where $L_{x}$ represents the contrast of a single pixel, and $L_{\text {ave }}$ is the average luminance value of the display]. Stimuli were presented on a uniform gray background such that mean luminance was constant across conditions; only stimulus contrast and size varied. The four conditions ("low-contrast small," "low-contrast large," "high-contrast small," and "high-contrast large") were presented separately in blocks of 150 trials; block order was counterbalanced across subjects. Stimuli drifted to the left or to the right at a rate of $2 \%$. Direction of motion was determined randomly for each trial and was equally probable in both directions.

Task. The task and analyses were conducted as previously reported (Tadin et al., 2003; Betts et al., 2005). Subjects pressed a button when they were ready to begin a block of trials. After $500 \mathrm{~ms}$, a flickering fixation dot appeared in the center of the screen, alternating between black and white at a rate of $2.5 \mathrm{~Hz}$ for $1.6 \mathrm{~s}$. After a delay of $500 \mathrm{~ms}$ during which the screen was blank, the grating appeared in the center of the screen and drifted in one direction for a specified duration (see below). The participant made an unspeeded two-alternative forced-choice response indicating the perceived direction of motion. After the response was made, there was a 500 ms delay until the next trial began.

Stimulus duration was varied using two interleaved staircases: a twodown, one-up staircase converging on the $71 \%$ correct duration threshold; and a four-down, one-up staircase converging on the $84 \%$ correct duration threshold. Initial stimulus duration for each condition was 150 ms; staircase implementation began after the second trial of the block. Initial step size for both staircases was $41.66 \mathrm{~ms}$; step size switched to $25.00 \mathrm{~ms}$ after three reversals and $8.33 \mathrm{~ms}$ after six reversals. Minimum stimulus duration was one frame $(8.33 \mathrm{~ms})$.

Practice tasks. Before beginning the experiment, subjects performed two short practice tasks. The first practice was designed to familiarize subjects with the basic task, and consisted of one block of 10 trials on an easily perceivable medium-contrast $(30 \%)$ medium-size $\left(2.5^{\circ}\right)$ stimulus. The second practice introduced subjects to the four stimulus size/contrast combinations presented in the main experiment. There was a single trial per condition, with the stimulus remaining on the screen for 5000 $\mathrm{ms}$. The goal of this second practice was to familiarize subjects with each stimulus, particularly the low-contrast small stimulus, which many subjects had initial difficulty perceiving. If subjects were unable to perceive a moving stimulus after the $5000 \mathrm{~ms}$ duration, practice for that condition was repeated until the subject reported successful motion perception.

Statistical analysis. Data from both staircases were combined, and accuracy on each trial was analyzed as a function of stimulus duration. For each subject and condition, stimulus durations were binned into $25 \mathrm{~ms}$ intervals, and the average accuracy was taken for each bin containing at least four samples. Psychometric functions were estimated by calculating the best-fitting Weibull function, and the duration threshold was defined by solving for the $77 \%$ correct point (Fig. 2). As mentioned above, for two control subjects and one recovered depressed patient, the Weibull fits for at least one condition did not converge on a $77 \%$ correct threshold, excluding these subjects from further analyses. Duration thresholds were submitted to a mixed-design repeated-measures ANOVA, with a 2 (size: small, large) $\times 2$ (contrast: low, high) $\times 2$ (group: patients, controls) design, where group was a between-subjects measure. Significant ANOVA interactions were further examined with post hoc one-way ANOVAs on each of the four duration threshold conditions, with group as the factor.

To quantify the degree of spatial suppression at high contrast and spatial summation at low contrast, we calculated separately for each contrast the suppression/summation index (Tadin et al., 2003; Betts et al., 2005): $\log _{10}$ (large stimulus threshold) $-\log _{10}$ (small stimulus threshold). Positive values reflect suppression, while negative values indicate summation. All analyses were conducted separately for the low and high contrast; one-way ANOVAs were conducted on the suppression/summation index with group as the between-subjects factor. For the patients, clinical measures of illness load (lifetime number of months spent acutely depressed) and recovery time (number of months since most recent MDD episode) were collected during clinical interviews with a psychiatrist; correlation analyses were run on these measures and the suppression and summation indices. If a significant correlation was found with suppression or summation index, correlations were subsequently run on the duration thresholds for the high- or low-contrast stimuli, respectively. All analyses were performed in Matlab (MathWorks), with additional statistical analysis conducted in SPSS version 16.

\section{Results}

\section{Motion discrimination thresholds}

Psychophysical motion discrimination thresholds for stimuli of high $(92 \%)$ and low $(2.8 \%)$ contrast and small $\left(0.7^{\circ}\right)$ and large $\left(5.0^{\circ}\right)$ size were compared for the two subject groups (Fig. 3). Collapsing across stimulus size and contrast, the recovered depressed and healthy control groups were equally able to perform the task. There was no main effect of group $\left(F_{(1,30)}=2.44, p=\right.$ $0.13)$, arguing against any generalized performance or motivation deficit in the patient group. Importantly, this eliminates a critical challenge present in most patient studies, including previous studies using this task (Betts et al., 2005; Tadin et al., 2006).

In both groups, performance was better for the low-contrast large stimuli relative to the low-contrast small stimuli, consistent with low-contrast spatial summation. On the other hand, both groups had more difficulty with the high-contrast large stimuli than the high-contrast small stimuli, demonstrating spatial suppression. This pattern was supported by a significant contrast $X$ 


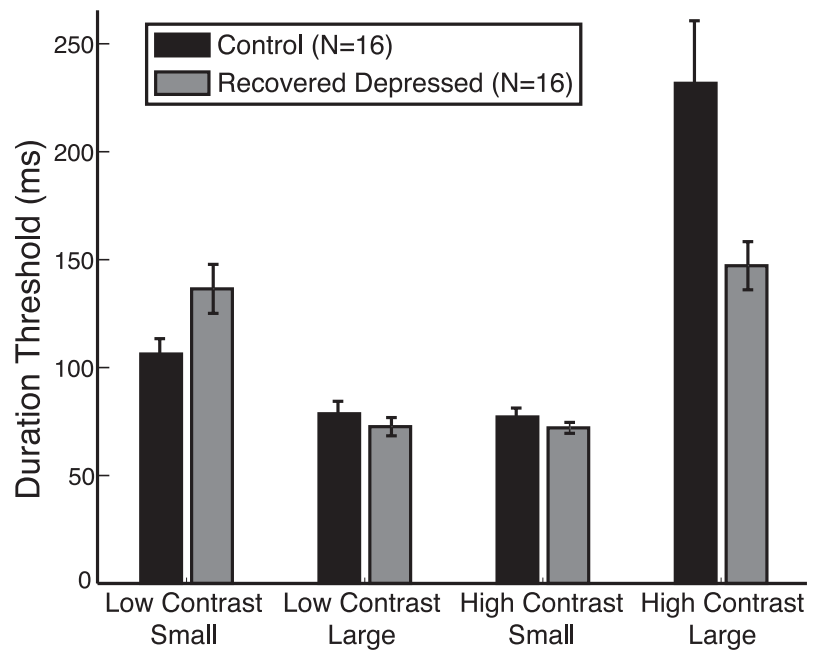

Figure 3. Motion discrimination thresholds. Mean motion discrimination thresholds are plotted for the recovered depressed group and control group for each of the four conditions: low-contrast small, low-contrast large, high-contrast small, and high-contrast large stimuli. Thresholds were calculated by fitting the duration/accuracy data for each subject and condition with psychometric Weibull functions and determining the duration required for $77 \%$ accurate performance (Fig. 2). Higher thresholds indicate that more time was needed to reliably discriminate the direction of motion for a particular condition, implying increased difficulty perceiving the motion stimulus. Error bars are SEM.

size interaction $\left(F_{(1,30)}=109.73, p<0.001\right)$, which was not significantly modulated by group $\left(F_{(1,30)}=1.99, p=0.17\right)$. However, group did modulate the main effects of size $\left(F_{(1,30)}=12.97\right.$, $p=0.001$ and $F_{(1,30)}=9.09, p=0.005$, main effect and interaction, respectively) and contrast $\left(F_{(1,30)}=17.67, p<0.001\right.$ and $F_{(1,30)}=12.70, p=0.001$, main effect and interaction, respectively).

Importantly, the MDD group showed less spatial suppression than the controls, supporting our hypothesis. The differences between the two groups were remarkably specific: the recovered depressed group performed worse than the controls on the lowcontrast small stimuli $\left(F_{(1,30)}=4.77, p=0.04\right)$ but better than the controls in the critical high-contrast large condition $\left(F_{(1,30)}=\right.$ 6.96, $p=0.01)$. On the other hand, both groups performed equally well on the easier low-contrast large and high-contrast small stimuli $\left(F<1\right.$, and $F_{(1,30)}=1.01, p=0.32$, respectively).

\section{Spatial suppression}

To quantify spatial suppression in the two groups, we calculated the suppression index as the log difference between thresholds for large and small high-contrast stimuli. The recovered depressed group showed significantly less suppression than the control group $\left(F_{(1,30)}=4.57, p=0.04\right)$; mean suppression index was 0.44 $(\mathrm{SD}=0.23)$ for the control group and $0.30(\mathrm{SD}=0.13)$ for the patient group. Furthermore, within the patient group, there was a significant inverse correlation of suppression index with illness load, measured as lifetime duration of depression in months $(r=$ $-0.51 ; p=0.04)$; patients who had spent the most time depressed exhibited the strongest differences (Fig. 4). This suggests that the pathophysiological process in MDD may be progressive and its functional consequences may accumulate over time. Although these deficits appear progressive, they may also be partially reversible during long periods of recovery. Subjects whose last MDD episode occurred more recently tended to show the weakest suppression, while patients who reported being recovered for longer tended to show more similar suppression to

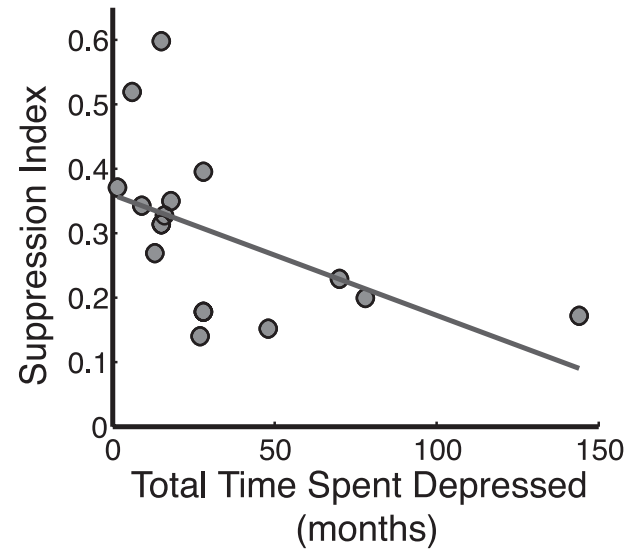

Figure 4. Suppression indices. High-contrast suppression indices are plotted for each individual recovered depressed patient as a function of the total number of lifetime months the patient reported having spent depressed, a measure of MDD illness load. Suppression index was calculated as $\log _{10}$ (high-contrast large threshold) - $\log _{10}$ (high-contrast small threshold); positive values indicate suppression. Scatter plots were fit with the linear best-fit regression line; $r=-0.51 ; p=0.04 ; n=16$. See Results for group means.

controls; this correlation approached significance $(r=0.45 ; p=$ 0.08). Although lifetime duration depressed and time since last depressed had opposite effects on the suppression index, the two clinical measures were not significantly correlated with each other $(r=0.15 ; p=0.58)$, suggesting that these are two independently varying processes affecting spatial suppression and presumably GABA levels.

To understand the source of these MDD effects on the suppression index, we examined how these clinical measures correlated with the two high-contrast duration thresholds that contributed to the index. Correlations with the high-contrast large threshold mimicked those of the suppression index: lifetime duration of depression showed a significant negative correlation $(r=-0.53 ; p=0.04)$, and time since last depressed showed a marginal positive correlation $(r=0.45 ; p=0.08)$. Neither clinical measure varied with the high-contrast small threshold $(r=$ $-0.15 ; p=0.58$, and $r=-0.27 ; p=0.31$, respectively), consistent with the lack of group difference for this condition. Thus, decreased spatial suppression in recovered depressed patients seems to be driven by an inability to suppress large, high-contrast stimuli, enabling the most severely affected patients to perform best at this particular task.

\section{Spatial summation}

To examine how MDD might also affect low-contrast spatial summation, we compared the log difference summation index for the low-contrast stimuli in a similar way. Since low-contrast large stimuli are easier to discriminate than low-contrast small stimuli, summation indices tend to be negative, with more negative indices reflecting enhanced summation. Recovered MDD patients demonstrated significantly enhanced spatial summation compared with controls $\left(F_{(1,30)}=7.43, p=0.01\right)$; mean summation index was $-0.13(\mathrm{SD}=0.15)$ for the control group and $-0.26(\mathrm{SD}=0.12)$ for the patient group. Summation index seemed to be strongly correlated with recovery time (Fig. 5); the more time that had passed since a patient's most recent episode, the weaker (and more like controls) was their summation index $(r=0.68 ; p=0.004)$. The time since last depressed measure was marginally correlated with low-contrast small threshold $(r=$ $-0.44 ; p=0.09$ ), but to a much lesser extent. Unlike the spatial suppression result, the amount of time a patient had spent de- 


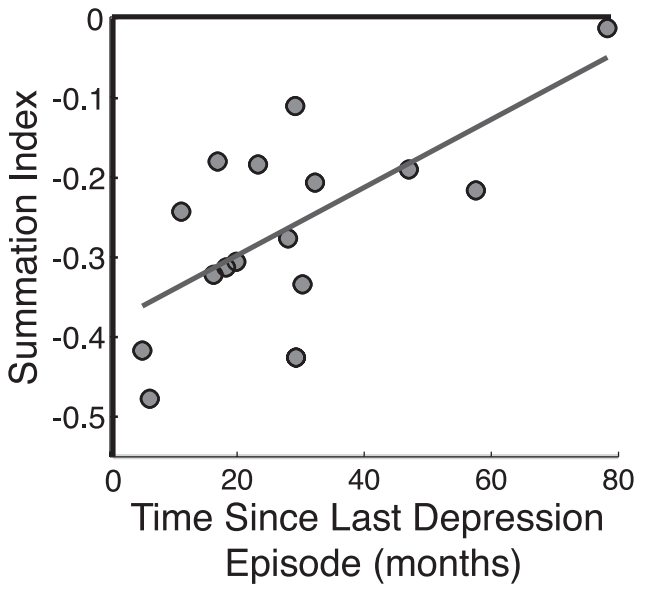

Figure 5. Summation indices. Low-contrast summation indices are plotted for each individual recovered depressed patient as a function of the number of months since the patient's most recent reported depression episode, a measure of MDD recovery duration. Summation index was calculated as $\log _{10}$ (low-contrast large threshold) $-\log _{10}$ (low-contrast small threshold); negative values indicate summation. Scatter plots were fit with the linear best-fit regression line; $r=0.68 ; p=0.004 ; n=16$. See Results for group means.

pressed did not significantly correlate with the summation index or any low-contrast threshold (all $r$ values $<0.4$, $p$ values $>0.15$ ).

\section{Discussion}

We report a novel functional consequence of MDD on visual processing. Fully recovered and asymptomatic medication-free subjects with recurrent MDD show decreased center-surround antagonism, manifesting as a behavioral reduction in highcontrast spatial suppression. This change in basic sensory processing worsens with increased duration of illness and persists long after mood symptoms have receded. The counterintuitive effect of this inhibitory deficit is an enhanced ability to perceive large high-contrast motion in MDD.

The visual system has not been traditionally considered a key component of the neural circuitry of MDD. However, studies that have included the occipital cortex in the analysis of patients with MDD have reported subtle but significant effects in occipital cortex, together with modulation in areas considered more central in the pathophysiology of MDD like corticolimbic regions (Nofzinger et al., 2005; Bhagwagar et al., 2006; Hasler et al., 2008; Moratti et al., 2008). Beyond these hints of occipital involvement in prior studies, the present results reveal a novel behavioral correlate of visual cortex functioning in MDD. Thus, the visual processing differences quantified using the current paradigm may represent a sensitive and critical biomarker of vulnerability to MDD. We suggest that these differences are related to a documented dysfunction of cortical GABA in this population (Sanacora et al., 1999, 2004; Bhagwagar et al., 2007, 2008). These differences could reflect a functional consequence of GABAergic dysfunction in the occipital cortex, a dysfunction of middle temporal cortical function, or an interaction between these posterior visual mechanisms. Although we did not directly measure GABA levels in this study and thus cannot differentiate between these and other underlying mechanisms, the highly specific pattern of results, in conjunction with previous reports linking GABA to both visual perception and MDD pathophysiology, supports a potential GABAergic mechanism for this novel behavioral consequence of MDD.

Enhanced processing of high-contrast large stimuli can be thought of as a benefit of reduced GABA, but this is not to imply that GABA deficiencies would be beneficial to the visual system in general. GABA-mediated center-surround antagonism is critical for successful execution of a number of perceptual abilities, including figure-ground segmentation, contour detection, and perception of three-dimensional structures (Allman et al., 1985; Gilbert, 1992; Kapadia et al., 1995; Lamme, 1995; Stemmler et al., 1995; Bradley et al., 1998; Fitzpatrick, 2000; Tadin and Lappin, 2005). We would expect MDD patients to have increased difficulty with these fine discrimination tasks - which may contribute to the patients' difficulty with the low-contrast small stimuliwhile similar difficulties at a cognitive level may underlie certain cognitive biases associated with MDD, such as an inability to inhibit negative or aversive memories (Davidson, 2002; Goeleven et al., 2006).

It should be noted that decreased spatial suppression in the current task was not driven by poorer overall performance in the patient group. Critically, there was no difference in the ability of patients with MDD to discriminate motion direction for the baseline high-contrast small stimulus compared with control subjects, and the patients performed better than the controls on the critical high-contrast large stimulus. This is in contrast to previous findings in clinical populations in which differences in suppression were driven by impaired baseline motion processing abilities (Tadin et al., 2006). The current study therefore represents an important advance over a common challenge in clinical research; that is, when decreased performance is reported for a clinical population, it can be unclear whether this should be interpreted as a result of neurobiological changes associated with the disorder, or as a secondary effect of decreased motivation or arousal. Our finding of enhanced performance in the patient group - combined with the fact that these perceptual effects were found in asymptomatic, unmedicated patients - provides strong evidence that these persistent functional consequences reflect the underlying pathophysiology of MDD and may be validated as an endophenotypic marker in future studies. Further studies will be required to validate the utility of spatial suppression as a predictor of vulnerability to future episodes of MDD or of response to treatment.

\section{References}

Allman J, Miezin F, McGuinness E (1985) Stimulus specific responses from beyond the classical receptive field: neurophysiological mechanisms for local-global comparisons in visual neurons. Annu Rev Neurosci 8:407-430.

Angelucci A, Bullier J (2003) Reaching beyond the classical receptive field of V1 neurons: horizontal or feedback axons? J Physiol Paris 97:141-154.

Bach M (1996) The Freiburg Visual Acuity Test-automatic measurement of visual acuity. Optom Vis Sci 73:49-53.

Betts LR, Taylor CP, Sekuler AB, Bennett PJ (2005) Aging reduces centersurround antagonism in visual motion processing. Neuron 45:361-366.

Bhagwagar Z, Wylezinska M, Taylor M, Jezzard P, Matthews PM, Cowen PJ (2004) Increased brain GABA concentrations following acute administration of a selective serotonin reuptake inhibitor. Am J Psychiatry 161:368-370.

Bhagwagar Z, Hinz R, Taylor M, Fancy S, Cowen P, Grasby P (2006) Increased 5-HT(2A) receptor binding in euthymic, medication-free patients recovered from depression: a positron emission study with [(11)C]MDL 100,907. Am J Psychiatry 163:1580-1587.

Bhagwagar Z, Wylezinska M, Jezzard P, Evans J, Ashworth F, Sule A, Matthews PM, Cowen PJ (2007) Reduction in occipital cortex gammaaminobutyric acid concentrations in medication-free recovered unipolar depressed and bipolar subjects. Biol Psychiatry 61:806-812.

Bhagwagar Z, Wylezinska M, Jezzard P, Evans J, Boorman E, M Matthews P, J Cowen P (2008) Low GABA concentrations in occipital cortex and anterior cingulate cortex in medication-free, recovered depressed patients. Int J Neuropsychopharmacol 11:255-260. 
Bradley DC, Chang GC, Andersen RA (1998) Encoding of threedimensional structure-from-motion by primate area MT neurons. Nature 392:714-717.

Brainard DH (1997) The psychophysics toolbox. Spat Vis 10:433-436.

Brambilla P, Perez J, Barale F, Schettini G, Soares JC (2003) GABAergic dysfunction in mood disorders. Mol Psychiatry 8:721-737, 715 .

Davidson RJ (2002) Anxiety and affective style: role of prefrontal cortex and amygdala. Biol Psychiatry 51:68-80.

Emrich HM, von Zerssen D, Kissling W, Möller HJ, Windorfer A (1980) Effect of sodium valproate on mania. The GABA-hypothesis of affective disorders. Arch Psychiatr Nervenkr 229:1-16.

First MB, Spitzer RL, Gibbon M, Williams JBM (1997) Structured clinical interview diagnostic (SCID) for DSM-IV axis I disorders-clinician version (SCID-CV).

Fitzpatrick D (2000) Seeing beyond the receptive field in primary visual cortex. Curr Opin Neurobiol 10:438-443.

Gilbert CD (1992) Horizontal integration and cortical dynamics. Neuron 9:1-13.

Goeleven E, De Raedt R, Baert S, Koster EH (2006) Deficient inhibition of emotional information in depression. J Affect Disord 93:149-157.

Hamilton M (1960) A rating scale for depression. J Neurol Neurosurg Psychiatry 23:56-62.

Hasler G, Fromm S, Carlson PJ, Luckenbaugh DA, Waldeck T, Geraci M, Roiser JP, Neumeister A, Meyers N, Charney DS, Drevets WC (2008) Neural response to catecholamine depletion in unmedicated subjects with major depressive disorder in remission and healthy subjects. Arch Gen Psychiatry 65:521-531.

Kalueff AV, Nutt DJ (2007) Role of GABA in anxiety and depression. Depress Anxiety 24:495-517.

Kapadia MK, Ito M, Gilbert CD, Westheimer G (1995) Improvement in visual sensitivity by changes in local context: parallel studies in human observers and in V1 of alert monkeys. Neuron 15:843-856.

Lamme VA (1995) The neurophysiology of figure-ground segregation in primary visual cortex. J Neurosci 15:1605-1615.

Lee S, Zhou ZJ (2006) The synaptic mechanism of direction selectivity in distal processes of starburst amacrine cells. Neuron 51:787-799.

Lloyd KG, Zivkovic B, Scatton B, Morselli PL, Bartholini G (1989) The gabaergic hypothesis of depression. Prog Neuropsychopharmacol Biol Psychiatry 13:341-351.

Moratti S, Rubio G, Campo P, Keil A, Ortiz T (2008) Hypofunction of right temporoparietal cortex during emotional arousal in depression. Arch Gen Psychiatry 65:532-541.

Nofzinger EA, Buysse DJ, Germain A, Price JC, Meltzer CC, Miewald JM, Kupfer DJ (2005) Alterations in regional cerebral glucose metabolism across waking and non-rapid eye movement sleep in depression. Arch Gen Psychiatry 62:387-396.

Pack CC, Hunter JN, Born RT (2005) Contrast dependence of suppressive influences in cortical area MT of alert macaque. J Neurophysiol 93:1809-1815.

Paffen CL, van der Smagt MJ, te Pas SF, Verstraten FA (2005) Centersurround inhibition and facilitation as a function of size and contrast at multiple levels of visual motion processing. J Vis 5:571-578.
Petty F (1995) GABA and mood disorders: a brief review and hypothesis. J Affect Disord 34:275-281.

Petty F, Kramer GL, Gullion CM, Rush AJ (1992) Low plasma gammaaminobutyric acid levels in male patients with depression. Biol Psychiatry 32:354-363.

Rush AJ, Kraemer HC, Sackeim HA, Fava M, Trivedi MH, Frank E, Ninan PT, Thase ME, Gelenberg AJ, Kupfer DJ, Regier DA, Rosenbaum JF, Ray O, Schatzberg AF, ACNP Task Force (2006) Report by the ACNP Task Force on response and remission in major depressive disorder. Neuropsychopharmacology 31:1841-1853.

Sanacora G, Mason GF, Rothman DL, Behar KL, Hyder F, Petroff OA, Berman RM, Charney DS, Krystal JH (1999) Reduced cortical gammaaminobutyric acid levels in depressed patients determined by proton magnetic resonance spectroscopy. Arch Gen Psychiatry 56:1043-1047.

Sanacora G, Mason GF, Rothman DL, Krystal JH (2002) Increased occipital cortex GABA concentrations in depressed patients after therapy with selective serotonin reuptake inhibitors. Am J Psychiatry 159:663-665.

Sanacora G, Mason GF, Rothman DL, Hyder F, Ciarcia JJ, Ostroff RB, Berman RM, Krystal JH (2003) Increased cortical GABA concentrations in depressed patients receiving ECT. Am J Psychiatry 160:577-579.

Sanacora G, Gueorguieva R, Epperson CN, Wu YT, Appel M, Rothman DL, Krystal JH, Mason GF (2004) Subtype-specific alterations of gammaaminobutyric acid and glutamate in patients with major depression. Arch Gen Psychiatry 61:705-713.

Schwabe L, Obermayer K, Angelucci A, Bressloff PC (2006) The role of feedback in shaping the extra-classical receptive field of cortical neurons: a recurrent network model. J Neurosci 26:9117-9129.

Seitz AR, Pilly PK, Pack CC (2008) Interactions between contrast and spatial displacement in visual motion processing. Curr Biol 18:R904-R906.

Shiah IS, Yatham LN (1998) GABA function in mood disorders: an update and critical review. Life Sci 63:1289-1303.

Stemmler M, Usher M, Niebur E (1995) Lateral interactions in primary visual cortex: a model bridging physiology and psychophysics. Science 269:1877-1880.

Tadin D, Lappin JS (2005) Linking psychophysics and physiology of centersurround interactions in visual motion processing. In: Seeing spatial form (Jenkin, RM, Harris, LR, eds), pp 279-314. Oxford: Oxford UP.

Tadin D, Lappin JS, Gilroy LA, Blake R (2003) Perceptual consequences of centre-surround antagonism in visual motion processing. Nature 424:312-315.

Tadin D, Kim J, Doop ML, Gibson C, Lappin JS, Blake R, Park S (2006) Weakened center-surround interactions in visual motion processing in schizophrenia. J Neurosci 26:11403-11412.

Tsumoto T, Eckart W, Creutzfeldt OD (1979) Modification of orientation sensitivity of cat visual cortex neurons by removal of GABA-mediated inhibition. Exp Brain Res 34:351-363.

Wilkinson GS (1993) WRAT-3: Wide Range Achievement Test administration manual. Wilmington, DE: Wide Range.

World Health Organization (2008) The global burden of disease: 2004 update. 